\title{
Serum microRNA miR-501-3p as a potential biomarker related to the progression of Alzheimer's disease
}

\author{
Norikazu Hara ${ }^{1}$, Masataka Kikuchi ${ }^{1}$, Akinori Miyashita ${ }^{1}$, Hiroyuki Hatsuta ${ }^{2}$, Yuko Saito ${ }^{3}$, Kensaku Kasuga ${ }^{1,4}$, \\ Shigeo Murayama ${ }^{2}$, Takeshi Ikeuchi ${ }^{1}$ and Ryozo Kuwano ${ }^{1,5,6^{*}}$
}

\begin{abstract}
MicroRNAs (miRNAs) are attractive molecules to utilize as one of the blood-based biomarkers for neurodegenerative disorders such as Alzheimer's disease (AD) because miRNAs are relatively stable in biofluid, including serum or plasma. To determine blood miRNA biomarkers for AD with next-generation sequencing genome-wide, we first surveyed 45 serum samples. These came from 27 AD patients and 18 controls (discovery set) that underwent autopsy within two weeks after their serum sampling and were neuropathologically diagnosed. We found that three miRNAs, hsa-miR-501-3p, hsa-let-7f-5p, and hsa-miR-26b-5p, were significantly deregulated between the $A D$ samples and the controls. The deregulation for hsa-miR-501-3p was further confirmed by quantitative reverse transcription polymerase chain reaction (PCR) in a validation set composed of 36 clinically diagnosed AD patients and 22 age-matched cognitively normal controls with a sensitivity and specificity of $53 \%$ and $100 \%$, respectively (area under the curve $=0.82$ ). Serum hsa-miR-501-3p levels were downregulated in AD patients, and its lower levels significantly correlated with lower Mini-Mental State Examination scores. Contrary to its serum levels, we found that hsa-miR-501-3p was remarkably upregulated in the same donors' AD brains obtained at autopsy from the discovery set. The hsa-miR-501-3p overexpression in cultured cells, which mimicked the hsa-miR-501-3p upregulation in the AD brains, induced significant downregulation of 128 genes that overrepresented the Gene Ontology terms, DNA replication, and the mitotic cell cycle. Our results suggest that hsa-miR-501-3p is a novel serum biomarker that presumably corresponds to pathological events occurring in AD brains.
\end{abstract}

Keywords: Alzheimer's disease, Blood-based biomarker, microRNA, miR-501-3p, Next-generation sequencing, Autopsied brain, Braak staging

\section{Introduction}

Alzheimer's disease (AD) is the most common form of dementia in the elderly and is characterized by two neuropathological hallmarks: extracellular senile plaques (SPs), composed of $\beta$-amyloid $(A \beta)$ peptides, and intraneuronal neurofibrillary tangles (NFTs) composed of abnormally hyperphosphorylated tau. To facilitate the clinical diagnosis of $\mathrm{AD}$, numerous studies have focused on biomarkers based on neuroimaging and molecules in

\footnotetext{
* Correspondence: ryosun@bri.niigata-u.ac.jp

${ }^{1}$ Department of Molecular Genetics, Bioresource Science Branch, Center for

Bioresources, Brain Research Institute, Niigata University, Niigata, Japan

${ }^{5}$ Asahigawaso Research Institute, Asahigawaso Medical-Welfare Center,

Okayama, Japan

Full list of author information is available at the end of the article
}

cerebrospinal fluid (CSF) and peripheral blood. For example, neuroimaging using positron emission tomography with the tracer carbon-11-labeled Pittsburgh compound $\mathrm{B}$ can detect $\mathrm{A} \beta$ deposits in the brains of living subjects. However, neuroimaging tests are expensive and available at only a limited number of laboratories. As a result, access to neuroimaging is confined to a small number of patients. Similarly, A $\beta$ and tau protein levels in CSF are well-established biomarkers with high accuracy for AD $[8,15]$, but CSF sampling by lumbar puncture is relatively invasive and requires skilled training. In contrast, blood sampling is less invasive and available at routine examinations. Once useful blood-based biomarkers are established, they allow physicians to screen a larger number of subjects. Recent studies have reported 
potential blood-based biomarkers for $\mathrm{AD}$ from various molecular species, such as protein, lipid, and nucleic acid [23].

MicroRNA (miRNA) is considered one of the candidates for blood-based biomarkers. miRNAs are $\sim 22 \mathrm{nt}$ small noncoding RNAs that bind to the 3 ' untranslated region of their target mRNAs to direct posttranscriptional repression of the target genes by forming the RNA-induced silencing complex, which leads to mRNA destabilization or translational inhibition [5, 6]. Some miRNAs are encapsulated in microvesicles, such as exosomes, and present as a relatively stable form in biofluid, including serum or plasma [39]. Recently, some sets of blood miRNAs have been shown to differentiate $\mathrm{AD}$ or mild cognitive impairment (MCI) from healthy controls [12, 21, 28, 32, 36, 42, 45]. For example, plasma levels of the miR-132 family (miR-128, miR-132, and miR-874) have distinguished MCI from age-matched controls with high accuracy (84-94\% sensitivity and 96-98\% specificity) [42]. In addition, alterations of miRNAs have also been observed in AD brains [20, 22, 41]. Hébert et al. have shown that in $\mathrm{AD}$ brains the miR-29a/b-1 cluster levels are decreased and correlated with the high BACE1 expression that leads to $A \beta$ generation, suggesting that these miRNAs directly regulate the BACE1 gene expression [22]. Despite the increasing evidence of miRNAs for AD pathology, their relationship between peripheral blood levels and brain levels, especially in the same individuals, is largely unexplored.

Here, we identified hsa-miR-501-3p as a novel serum miRNA biomarker that was downregulated in $\mathrm{AD}$ patients. We measured hsa-miR-501-3p levels in the same donors' brains that were obtained at autopsy within two weeks after their serum sampling and found remarkable upregulation of hsa-miR-501-3p. To elucidate the effects of the hsa-miR-501-3p upregulation in the AD brains, we performed hsa-miR-501-3p overexpression analysis in cultured cells.

\section{Materials and methods Subjects}

We prepared two independent sample sets to discover and validate potential serum miRNA biomarkers for AD. The discovery set, ROW, consisted of samples obtained from the Japanese Brain Bank Network for Neuroscience Research (JBBNNR), where all autopsied brains are systematically diagnosed with Braak staging $[10,11]$ and stored at $-80{ }^{\circ} \mathrm{C}$. We used serum that was collected from donors in routine blood tests within two weeks before their death. The temporal cortex tissues from the same donors were dissected by experts at JBBNNR and stored at $-80^{\circ} \mathrm{C}$ until use.

The validation set, NIG, was composed of clinically diagnosed subjects from Niigata University Medical and
Dental Hospital. The diagnosis of AD was made by the criteria of the National Institute of Neurological and Communicative Diseases and the Stroke-Alzheimer's Disease and Related Disorders Association [38]. Serum was collected from the subjects during the clinical follow-up period in the outpatient department and stored at $-80{ }^{\circ} \mathrm{C}$ until analysis.

\section{APOE genotyping}

Isolation of genomic DNA from brain or blood samples and $A P O E$ genotyping were performed as previously described [29].

\section{RNA extraction}

Cell-free RNA was isolated from $200 \mu \mathrm{l}$ of serum with miRNeasy Serum/Plasma Kit (QIAGEN, Hilden, Germany). For quantitative reverse transcription PCR (qRT-PCR) assay of the NIG validation set, $5.6 \times 10^{8}$ copies of synthetic cel-miR-39-3p (miRNeasy Serum/ Plasma Spike-In Control; QIAGEN) were added to each serum sample to normalize and monitor extraction efficiency. Finally, serum RNA was eluted in $14 \mu \mathrm{l}$ of RNase-free water.

Total RNA was isolated from a small piece of the frozen temporal cortex tissue or cultured cells with TRIzol Plus RNA Purification Kit (Thermo Fisher Scientific, Waltham, MA, USA). The isolated total RNA was loaded on the Agilent 2100 Bioanalyzer instrument (Agilent Technologies, Santa Clara, CA, USA) to determine the value of the RNA integrity number (RIN), which indicates the quality of mRNA transcripts and ranges from 1 (totally degraded) to 10 (intact).

\section{Small RNA sequencing}

We prepared small RNA sequencing libraries with the TruSeq Small RNA Sample Prep Kit (Illumina, San Diego, CA, USA), using $1 \mu \mathrm{g}$ of total RNA or $5 \mu \mathrm{l}$ of the $14 \mu \mathrm{l}$ purified serum RNA as input. Each library was sequenced on Illumina Genome Analyzer IIx (GAIIx), and more than 20,000,000 single-end reads were obtained from each library. Adapter sequences were trimmed from the sequenced reads with cutadapt [37], and then the reads were mapped using bowtie [30] against the human genome build hg19. The number of mapped reads was counted using HTSeq [3] in each miRNA from miRBase release 20 [26]. The raw read counts were normalized by the median-of-ratios method implemented in DESeq2 [35]. To remove low-abundance miRNAs, we filtered out miRNAs with $<50$ mean normalized read counts across all samples. We used DESeq2 [35] to identify deregulated miRNAs between AD and controls. We also performed differential miRNA expression analysis with DESeq2's design formula including the additional covariates, age, sex, $A P O E$ genotype, and hemolysis 
ratio or RIN, to adjust for these variables. $P$-values were calculated by the Wald test and adjusted by the BenjaminiHochberg procedure [7] for multiple testing correction. We considered miRNAs with $<0.05$ adjusted $p$-value $(5 \%$ false discovery rate (FDR)) statistically significant.

\section{Evaluation of hemolysis}

To detect the degree of hemolysis during serum preparation, free hemoglobin levels were measured spectrophotometrically. $12 \mu \mathrm{l}$ of serum was diluted with $48 \mu \mathrm{l}$ of phosphate buffered saline and then used to determine the ratio of the absorbance at $414 \mathrm{~nm}$ and $375 \mathrm{~nm}$, hemolysis ratio $\left(A_{414 / 375}\right)$, whose higher values show hemolyzed samples $[25,49]$. We removed serum samples with $>2$ hemolysis ratio from the NIG validation set.

\section{qRT-PCR}

Candidate serum miRNAs were validated by qRT-PCR using TaqMan MicroRNA Assays (Thermo Fisher Scientific). $5 \mu$ of serum RNA was reverse transcribed to cDNA with the TaqMan MicroRNA Reverse Transcription kit using an RT primer pool containing three target miRNA primers (hsa-miR-501-3p, hsa-let-7f-5p, and hsa-miR-26b-5p) and two normalization miRNA primers (cel-miR-39-3p and hsa-miR-451a) at a final concentration of $0.2 \times$ each in a reaction volume of $15 \mu \mathrm{l} .5 \mu \mathrm{l}$ of the RT product was preamplified for 12 cycles with TaqMan PreAmp Master Mix using a preamplification primer pool containing those five miRNA primers at a final concentration of $0.03 \times$ each in a reaction volume of $25 \mu \mathrm{l}$. The preamplified product was diluted by adding $100 \mu \mathrm{l}$ of $0.1 \times \mathrm{TE}$. Using $5 \mu \mathrm{l}$ of the diluted product, $\mathrm{qPCR}$ was performed in triplicate in a reaction volume of $20 \mu \mathrm{l}$ in a 384-well plate on an ABI PRISM 7900HT instrument with TaqMan MicroRNA Assays for each miRNA. Relative expression levels of miRNAs were calculated by the $2^{-\Delta \Delta C}$ T method [34] using cel-miR-39-3p (spike-in) and hsa-miR-451a (endogenous control) for normalization.

\section{Cell culture and miRNA transfection}

A human neuroblastoma cell line of SH-SY5Y cells was cultured in a 1:1 mixture of minimum essential medium and Ham's F-12 nutrient mixture supplemented with $10 \%$ fetal bovine serum and $1 \%$ penicillin-streptomycin in a $\mathrm{CO}_{2}$ incubator. One day after seeding, the cells were transfected with either hsa-miR-501-3p mimic (MC12927; Thermo Fisher Scientific) or scramble control (mirVana ${ }^{\mathrm{Tm}}$ miRNA Mimic, Negative Control \#1; Thermo Fisher Scientific) at $50 \mathrm{nM}$ final concentration using NeuroMag (OZ Biosciences, San Diego, CA, USA). After $24 \mathrm{~h}$, the cells were treated with the TRIzol reagent and stored at $-80{ }^{\circ} \mathrm{C}$ until RNA extraction.
mRNA sequencing and gene ontology analysis

mRNA sequencing libraries were prepared using TruSeq RNA Sample Prep Kit v2 (Illumina) with 500 ng of total RNA as input. Each library was sequenced on GAIIx, and approximately 40,000,000 of 75 bp single-end reads were obtained from each library. The sequenced reads were mapped using STAR [18] against the human genome (hg19). We used DESeq2 [35] to perform differential gene expression analysis and considered genes with $<0.05$ adjusted $p$-value (5\% FDR) statistically significant. We used DAVID $6.8[16,17]$ to perform Gene Ontology enrichment analysis on significantly differentially expressed genes, focusing on Gene Ontology category GOTERM_BP_DIRECT. We set the statistical significance threshold at 0.05 (5\% FDR).

\section{Results}

Discovery of candidate serum miRNA biomarkers for AD To prepare the ROW discovery set, we deliberately selected cases whose autopsied brain and serum samples alike were archived in JBBNNR. According to Braak staging $[10,11]$, we defined cases with Braak NFT stages IV through VI and Braak amyloid stage $\mathrm{C}$ as $\mathrm{AD}$, and cases with Braak NFT stages 0 through II and Braak amyloid stage 0 or A as controls (Additional file 1: Figure S1) [40]. These definitions of $\mathrm{AD}$ and controls prevented the study sample from including transient forms of the disease (e.g., cases with Braak NFT stage III) so that we could distinctly detect the difference between patients with typical $A D$ and controls without obvious pathological findings. As a result, 27 neuropathologically diagnosed AD patients and 18 control subjects were selected. The ROW discovery set had significant differences $(P<0.05)$ in age at death, sex, brain weight, and RIN between AD and controls (Table 1).

We isolated cell-free RNA from serum collected from the donors within two weeks before their death. The serum RNA was analyzed by high-throughput nextgeneration sequencing (NGS) to compare miRNA levels between $\mathrm{AD}$ and controls. We filtered out low-abundance miRNAs by mean normalized read counts. Of the remaining 148 miRNAs, 3 miRNAs had significant changes in expression level: hsa-miR-501-3p (adjusted $P=0.002, \log 2$ fold change $=-1.58$ ), hsa-let-7f-5p (adjusted $P=0.026, \log 2$ fold change $=1.00$ ), and hsamiR-26b-5p (adjusted $P=0.026, \log 2$ fold change $=$ 0.93). In the $A D$ patients, hsa-miR-501-3p was downregulated and the other two were upregulated (Fig. 1a). In particular, hsa-miR-501-3p still had a significant change in expression level after adjusting for age, sex, $A P O E$ genotype, and hemolysis ratio (adjusted $P=$ $0.004, \log 2$ fold change $=-2.07$; Additional file 2: Table S1). We added the hemolysis ratio of serum samples to those covariates because hemolysis could affect some miRNAs' levels [25]. 
Table 1 Demographics of the two sample sets in this study

\begin{tabular}{|c|c|c|c|}
\hline & $\mathrm{CT}$ & $A D$ & $P$-value \\
\hline \multicolumn{4}{|l|}{ ROW discovery set } \\
\hline $\mathrm{N}$ & 18 & 27 & - \\
\hline$A A D, y r$ & $76.3 \pm 7.1$ & $84.5 \pm 8.0$ & $0.001^{a}$ \\
\hline Gender, \% (F: M) & $27.8: 72.2$ & $63.0: 37.0$ & $0.033^{b}$ \\
\hline PMI, hr & $9.6 \pm 9.4$ & $13.4 \pm 11.6$ & $0.158^{a}$ \\
\hline BW, g & $1268 \pm 127$ & $1156 \pm 120$ & $0.003^{\mathrm{a}}$ \\
\hline \multicolumn{4}{|l|}{ RIN } \\
\hline $\mathrm{TC}$ & $7.9 \pm 0.8$ & $7.1 \pm 1.2$ & $0.006^{\mathrm{a}}$ \\
\hline \multicolumn{4}{|l|}{ Hemolysis ratio } \\
\hline Serum & $1.9 \pm 0.6$ & $1.7 \pm 0.5$ & $0.105^{a}$ \\
\hline \multicolumn{4}{|l|}{$A P O E$} \\
\hline $\begin{array}{l}\text { Genotype, \% } \\
\left(\varepsilon 3^{*} 3: \varepsilon 3^{*} 4: \varepsilon 4^{*} 4\right)\end{array}$ & $55.6: 44.4: 0.0$ & $44.4: 25.9: 29.6$ & $0.550^{c}$ \\
\hline Allele, \% (દ3 : ع4) & $77.8: 22.2$ & $57.4: 42.6$ & $0.069^{c}$ \\
\hline \multicolumn{4}{|l|}{ NIG validation set } \\
\hline $\mathrm{N}$ & 22 & 36 & - \\
\hline$A A E, y r$ & $73.7 \pm 8.4$ & $74.7 \pm 7.3$ & $0.556^{\mathrm{a}}$ \\
\hline Gender, \% (F: M) & $80.0: 20.0$ & $63.9: 36.1$ & $0.333^{b}$ \\
\hline MMSE & $29.3 \pm 0.7$ & $19.3 \pm 5.4$ & 4.8.E-08 \\
\hline \multicolumn{4}{|l|}{ Hemolysis ratio } \\
\hline Serum & $1.3 \pm 0.2$ & $1.3 \pm 0.2$ & $0.316^{a}$ \\
\hline \multicolumn{4}{|l|}{$A P O E$} \\
\hline $\begin{array}{l}\text { Genotype, \% } \\
\left(\varepsilon 3^{*} 3: \varepsilon 3^{*} 4\right)\end{array}$ & $85.7: 14.3$ & $55.6: 44.4$ & $0.056^{c}$ \\
\hline Allele, \% (ع3 : ع4) & $92.9: 7.1$ & $77.8: 22.2$ & $0.090^{c}$ \\
\hline
\end{tabular}

Abbreviations: $A A D$ age at death, $A A E$ age at examination, $A D$ Alzheimer's disease, $A P O E$ apolipoprotein $\mathrm{E}, B W$ brain weight, $C T$ control, $F$ female, $g$ grams, $h r$ hours, $M$ male, MMSE mini-mental state examination, $P M I$ postmortem interval, RIN RNA integrity number, $T C$ temporal cortex, yr years

${ }^{a}$ Calculated by Mann-Whitney $U$-test between AD and CT

${ }^{\mathrm{b} C a l c u l a t e d}$ by Fisher's exact test for gender distribution

${ }^{c}$ Calculated by Fisher's exact test for $A P O E \varepsilon 4$ allele carrier status ( $\varepsilon 4$ carrier and $\varepsilon 4$ non-carrier)

Data are presented as the mean \pm standard deviation

\section{Validation of candidate serum miRNAs}

To validate the three candidate serum miRNAs, we prepared sample set NIG, which was composed of 36 clinically diagnosed AD patients and 22 age-matched cognitively normal controls (Table 1). Serum was collected from those subjects and examined for the degree of hemolysis in the same way as the ROW discovery set. To evaluate more strictly the miRNA levels, we excluded the serum samples with a $>2$ hemolysis ratio from the NIG validation set according to Zanutto et al. [49]. Cellfree RNA was isolated from the nonhemolyzed serum samples and analyzed by qRT-PCR to quantify the miRNA levels. The qRT-PCR analysis revealed that hsa-miR-501$3 p$ had a significant, consistent change in expression level $(P=0.00002, \log 2$ fold change $=-0.54$; Fig. $1 \mathrm{~b})$, whereas hsa-miR-26b-5p showed marginal significance $(P=0.066$, $\log 2$ fold change $=0.20$; Fig. 1b). Furthermore, serum hsamiR-501-3p levels significantly correlated with MiniMental State Examination scores $(r=0.375, P=0.007$; Fig. 1c). The receiver-operating characteristic curve of hsa-miR-501-3p showed 53\% sensitivity and 100\% specificity (area under the curve $=0.82$; Fig. $1 \mathrm{~d}$ ). These results suggest that hsa-miR-501-3p could be a novel serum biomarker for clinical diagnosis of AD.

\section{Deregulated hsa-miR-501-3p in AD brains}

To investigate whether serum hsa-miR-501-3p levels had an association with brain levels, we measured hsa-miR501-3p levels in the same donors' brains that were obtained at autopsy within two weeks after their serum sampling. We used those autopsied brains in the ROW discovery set and isolated total RNA from their temporal cortex, a brain region that is severely affected in the late stages of AD progression [11]. The total RNA was analyzed by NGS to compare miRNA levels between AD and controls. The NGS analysis revealed that 187 of $472 \mathrm{miR}-$ NAs were significantly deregulated (adjusted $P<0.05$; Additional file 2: Table S2). This included hsa-miR-501$3 \mathrm{p}$, which was prominently upregulated in the AD brains and had the third lowest $p$-value (adjusted $P=2.9$.E-08, $\log 2$ fold change $=1.98$, rank $=3$; Fig. $2 \mathrm{a}$ and Additional file 2: Table S2). We found that hsa-miR-501-3p still had a significant change in expression level after adjusting for age, sex, $A P O E$ genotype, and RIN (adjusted $P=0.0008$, $\log 2$ fold change $=1.33$, rank $=25$; Additional file 2: Table S3). Moreover, hsa-miR-501-3p levels significantly correlated with Braak NFT stages (Fig. 2b). As AD pathology progressed, brain hsa-miR-501-3p levels increased $(r=0.436, P=0.003)$ while its serum levels decreased $(r=-0.355, P=0.017)$. We also observed the negative correlation between brain hsa-miR-501$3 p$ levels and serum hsa-miR-501-3p levels in the same individuals, although it was not significant $(r=$ $-0.17, P=0.25$; Fig. 2c). Collectively, hsa-miR-501-3p was deregulated in the brain as well as in the serum of the same individuals during AD progression.

\section{Functional assays of hsa-miR-501-3p in cultured cells}

To elucidate the effects of the hsa-miR-501-3p upregulation in AD brains, we transfected synthetic hsa-miR501-3p into SH-SY5Y cells. Total RNA isolated from the cultured cells was analyzed by NGS to identify genes that are regulated by hsa-miR-501-3p. Overexpression of hsa-miR-501-3p in SH-SY5Y cells resulted in significant changes in the expression levels of 208 genes in comparison to the scramble control (adjusted $P<0.05$; Additional file 2: Table S4). Of the 208 significant genes, 128 were downregulated in hsa-miR-501-3p overexpression. To investigate whether hsa-miR-501-3p directly represses the gene expression of those downregulated 
a

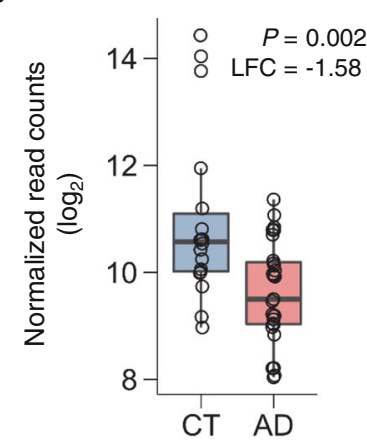

hsa-let-7f-5p

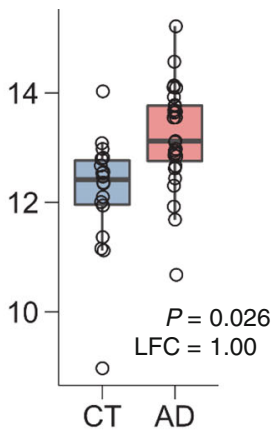

hsa-let-7f-5p

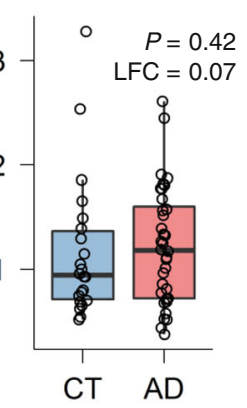

hsa-miR-26b-5p

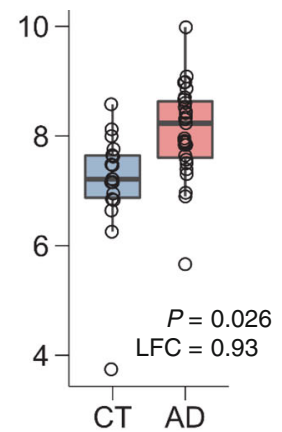

b

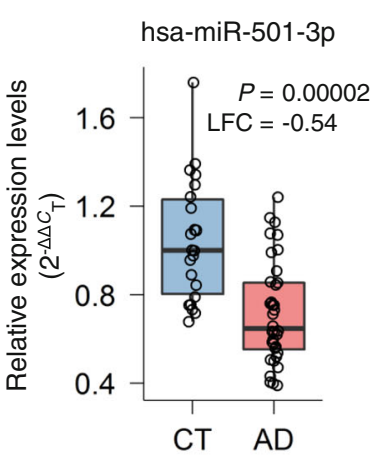

c

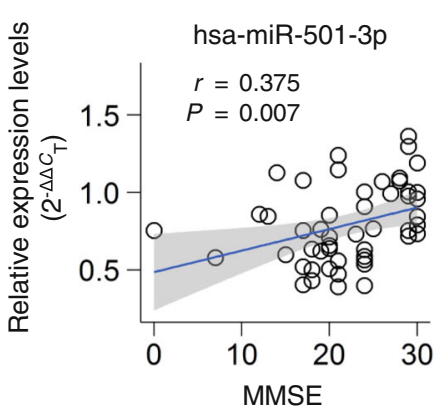

hsa-miR-26b-5p

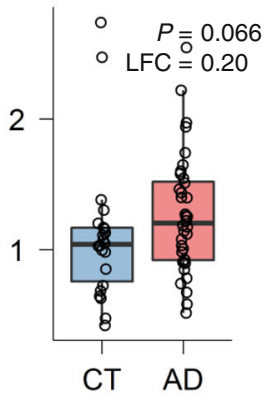

d

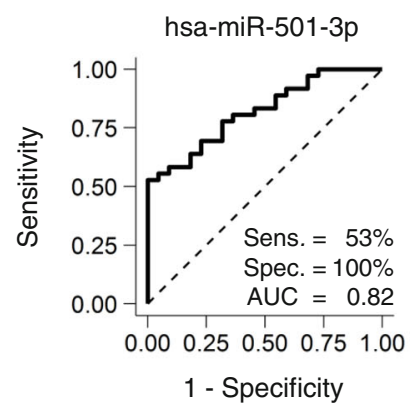

Fig. 1 A novel serum miRNA biomarker hsa-miR-501-3p that differentiates patients with Alzheimer's disease (AD) from controls (CT). a Serum miRNA levels of the three candidates significantly deregulated between AD and controls in the ROW discovery set. Normalized read counts from next-generation sequencing data were converted to a log 2 scale and plotted against the disease status. The lower hinge, the line in the middle, and the upper hinge of the box plot show the 25th percentile, 50th percentile (median), and 75th percentile, respectively. The lower whisker extends from the lower hinge to the lowest value that is within 1.5 times the interquartile range (IQR) of the hinge. The upper whisker extends from the upper hinge to the highest value that is within 1.5 times the IQR. LFC: log2 fold change; P: $p$-value adjusted by the Benjamini-Hochberg procedure for multiple testing correction. $\mathbf{b}$ Three candidates' serum miRNA levels quantified by quantitative reverse transcription PCR (qRT-PCR) in the NIG validation set. Relative expression levels of the miRNAs were calculated using the $2^{-\Delta \Delta C}$ T method with cel-miR-39-3p (spike-in) and hsa-miR-451a (endogenous control) for normalization and were plotted against the disease status. P-values were computed using the Mann-Whitney U-test between AD and controls. Box plots display the distributions of data in the same way as in Fig. 1a. LFC: log2 fold change. c Significant positive correlation between MiniMental State Examination (MMSE) scores and serum hsa-miR-501-3p levels in the NIG validation set. Relative expression levels from the qRT-PCR data were plotted against MMSE. $r$ shows the Spearman rank correlation coefficient. The blue line shows a linear regression line, and a shaded gray area around the line represents 95\% confidence intervals. $\mathbf{d}$ The receiver-operating characteristic (ROC) curve of serum hsa-miR-501-3p in the NIG validation set. The ROC curve analysis showed 53\% sensitivity and 100\% specificity. The area under the ROC curve (AUC) was 0.82

genes we used TargetScan 7.1, a database of predictive targets of each miRNA [2]. Of the 128 downregulated genes, 123 were annotated by TargetScan 7.1, which predicted that hsa-miR-501-3p bound to 71 of the 123 genes $(71 / 123(58 \%))$. This rate of predictive targets of
hsa-miR-501-3p was significantly higher than that of all the genes tested and annotated by TargetScan 7.1 (1788/ 11483 (16\%), Fisher's exact test, $P<9.7 . E-16)$, supporting that hsa-miR-501-3p overexpression successfully induced alteration of the gene expression in the cultured 


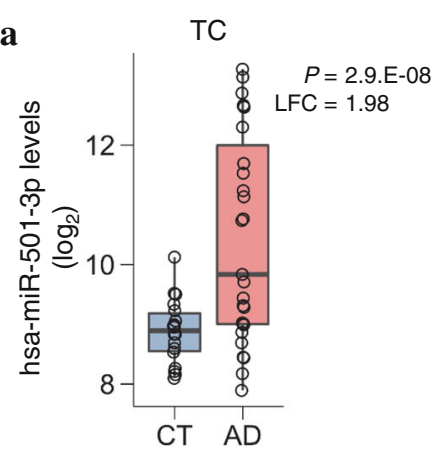

b
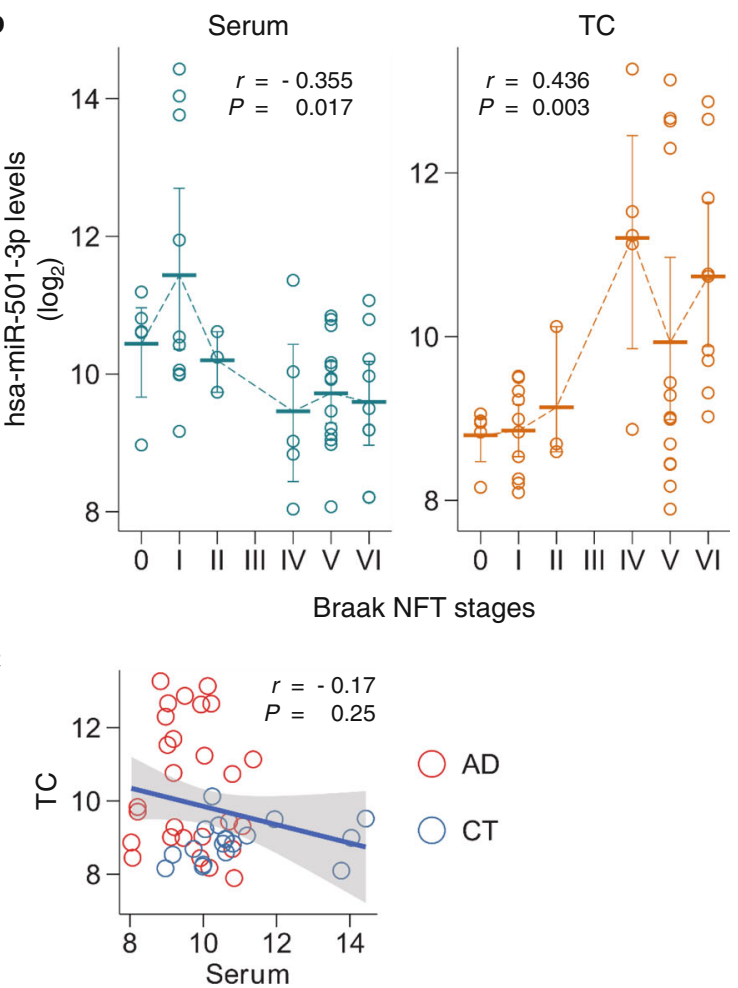

Fig. 2 Deregulated hsa-miR-501-3p not only in the serum but also in the brain of the same individuals over the course of Alzheimer's disease (AD) progression. a Brain hsa-miR-501-3p levels prominently upregulated in the AD brains of the ROW discovery set. The levels of hsa-miR-501-3p in the temporal cortex were analyzed using next-generation sequencing (NGS). Normalized read counts from the NGS data were converted to a log2 scale and plotted against the disease status. Box plots display the distributions of data in the same way as in Fig. 1a. CT: control; LFC: log2 fold change; P: $p$-value adjusted by the Benjamini-Hochberg procedure for multiple testing correction; TC: temporal cortex. $\mathbf{b}$ Significant correlation of either serum hsa-miR-501-3p levels or brain hsa-miR-501-3p levels with Braak NFT stages in the ROW discovery set. While serum hsa-miR-501-3p levels negatively correlated with Braak NFT stages (Spearman's $r=-0.355, P=0.017$ ), brain hsa-miR-501-3p levels positively correlated with Braak NFT stages (Spearman's $r=0.436, P=0.003$ ). Normalized read counts from the NGS data were converted to a log2 scale and plotted against Braak NFT stages. Each thick bar shows the mean value of hsa-miR-501-3p levels in a Braak NFT stage. Each error bar shows a 95\% confidence interval of hsa-miR-501-3p levels in a Braak NFT stage. TC: temporal cortex. c Negative correlation between serum hsa-miR-501-3p levels and brain hsa-miR-501-3p levels in the same individuals of the ROW discovery set. The levels of hsa-miR-501-3p between serum and brain of the same individuals were insignificantly yet negatively correlated (Spearman's $r=-0.17, P=0.25$ ). Normalized read counts of hsa-miR-501-3p levels were converted to a log2 scale and plotted. The $x$-axis and $y$-axis show serum hsa-miR-501-3p levels and brain hsa-miR-501-3p levels, respectively. The blue line shows a linear regression line, and a shaded gray area around the line represents 95\% confidence intervals. Red circles and blue circles show the hsa-miR-501-3p levels of AD patients and control subjects, respectively. CT: control; TC: temporal cortex

cells. To uncover the biological function of the genes that were altered in hsa-miR-501-3p overexpression, we used DAVID $6.8[16,17]$ to perform Gene Ontology enrichment analysis on the 208 significant genes. DAVID
6.8 revealed that the set of the 128 downregulated genes significantly overrepresented some terms of Gene Ontology, such as DNA replication and mitotic cell cycle (Additional file 2: Table S5). No significant enrichment 
was observed in the remaining 80 upregulated genes (Additional file 2: Table S6).

\section{Discussion}

There is a significant link between the developments of SPs and NFTs in the brain and the alterations of $A \beta$ and tau protein levels in CSF, thus making CSF levels useful biomarkers that facilitate clinical diagnosis of AD. In this study, we found that serum hsa-miR-501-3p levels were downregulated while brain levels were prominently upregulated in the $\mathrm{AD}$ patients of the ROW discovery set. Both serum and brain hsa-miR-501-3p levels significantly correlated with Braak NFT stages, suggesting that those hsa-miR-501-3p levels are related to the disease progression. In addition, the alteration of serum hsamiR-501-3p was replicated in the NIG validation set composed of clinical samples. These results indicate that serum hsa-miR-501-3p is a plausible biomarker for AD and its serum levels presumably correspond to its brain levels, which links to $\mathrm{AD}$ pathology. There was up to a two-week time lag between the serum sampling and brain autopsy in the ROW discovery set, but a time of two weeks lends little difference according to the gradual progression of $\mathrm{AD}$ pathology over years. Therefore, we consider our serum and brain samples in the ROW discovery set to be collected from each donor at approximately the same time point.

To our knowledge, alteration of hsa-miR-501-3p in peripheral blood has never been reported in preceding AD biomarker studies. This is probably due to technical differences, including different methods of RNA extraction [14, 33], different quantitative techniques [47], and different sources as input such as plasma, serum, and whole blood. For instance, miRNA contents from blood cells are much greater than those from cell-free plasma or serum [47]. Hence, whole blood samples or highly hemolyzed samples including contaminants of blood cells may result in outcomes different from serum or plasma samples. In fact, we discovered hsa-let-7f-5p and hsa-miR-26b-5p that were upregulated in serum, whereas Leidinger et al. [32] have reported decreased levels in whole blood from $\mathrm{AD}$ patients. The different starting materials, namely serum or whole blood, may lead to the inconsistent results. Consequently, it is vital to follow a standardized protocol stringently to achieve a consensus on miRNA biomarkers.

Few studies have described the deregulation of hsamiR-501-3p in AD brains as well as peripheral blood. This may be attributed to the difference in Braak NFT stages of autopsied brain samples. Our NGS analysis in the ROW discovery set showed that brain hsa-miR-501$3 p$ levels did not continuously rise over the Braak NFT stages; its levels temporarily dropped in Braak NFT stage V (Fig. 2b). Thus, which Braak NFT stages researchers define for $\mathrm{AD}$ and controls can determine the outcome of a study. However, the NGS analysis revealed the deregulation of miRNAs that other studies have described (e.g., miR-107 [46], miR-125b [4], and the miR-132/212 cluster [31, 43, 48]). As Lau et al. have shown [31], further work is needed to uncover the pattern of miRNA levels over the Braak NFT stages with dozens of brain samples from different cortical regions in each Braak NFT stage.

Although it remains elusive as to how the upregulation of hsa-miR-501-3p in the brain has an effect on $A D$ pathogenesis, our results indicate a possibility that hsamiR-501-3p upregulation could cause alterations in the cell cycle in AD brains. We found that hsa-miR-501-3p overexpression in cultured cells induced the downregulation of 128 genes that were significantly enriched in the biological processes of DNA replication and mitotic cell cycle (Additional file 2: Table S5). Accumulating evidence indicates that inappropriate cell cycle reentry in postmitotic neurons, which leads to apoptotic cell death, is an early sign that precedes the developments of SPs and NFTs in AD brains [1, 9, 13, 19, 27, 44]. Alternatively, $\mathrm{Hu}$ et al. [24] have recently demonstrated in rat brains that miR-501-3p mediates the activity-dependent regulation of the expression of the AMPA receptor subunit GluA1 in dendrites, suggesting that miR-501-3p contributes to synaptic plasticity related to cognitive functions, including learning and memory.

\section{Conclusions}

In summary, we discovered that hsa-miR-501-3p is strongly suggested as a novel serum biomarker for AD. Our study associated the downregulation of serum hsamiR-501-3p levels with its remarkable upregulation in AD brains, which may be involved in the pathogenesis of AD through an aberrant neuronal cell cycle. Our study suggests that serum hsa-miR-501-3p is a novel indicator that presumably reflects the progression of $\mathrm{AD}$.

\section{Additional files}

\footnotetext{
Additional file 1: Figure S1. This study's definitions of patients with Alzheimer's disease (AD) and controls on the basis of Braak staging in the ROW discovery set. (PDF $186 \mathrm{~kb}$ )

Additional file 2: Table S1. Top 20 deregulated serum miRNAs that were identified by NGS in the ROW discovery set after adjusting for age, sex, APOE genotype, and hemolysis ratio. Table S2. Significantly deregulated miRNAs that were identified by NGS in the temporal cortex of the ROW discovery set. Table S3. Significantly deregulated miRNAs that were identified by NGS in the temporal cortex of the ROW discovery set after adjusting for age, sex, APOE genotype, and RIN. Table S4. Significantly differentially expressed genes that were identified by NGS in hsa-miR-501$3 p$ overexpression in cultured cells. Table S5. Gene Ontology enrichment analysis on the significantly downregulated genes in hsa-miR-501-3p overexpression in cultured cells. Table S6. Gene Ontology enrichment analysis on the significantly upregulated genes in hsa-miR-501-3p overexpression in cultured cells. (XLS $172 \mathrm{~kb}$ )
} 


\section{Abbreviations}

AD: Alzheimer's disease; A $\beta$ : $\beta$-amyloid; CSF: Cerebrospinal fluid; FDR: False discovery rate; GAllx: Genome Analyzer IIx; JBBNNR: Japanese Brain Bank Network for Neuroscience Research; MCl: Mild cognitive impairment; miRNA: microRNA; NFT: Neurofibrillary tangle; NGS: Next-generation sequencing; qRT-PCR: Quantitative reverse transcription PCR; RIN: RNA integrity number; SP: Senile plaque

\section{Acknowledgments}

We gratefully acknowledge the efforts of our clinical and laboratory staff. We thank patients and their families, whose help and participation made this work possible.

\section{Funding}

This study was supported by (1) the "Japanese Alzheimer's Disease Neuroimaging Initiative" of the New Energy and Industrial Technology Development Organization (NEDO) (Grant number P12009), Japan (RK); (2) MEXT/JSPS KAKENHI, Grant-in-Aid for Scientific Research on Priority Areas, Scientific Research (B) and Challenging Exploratory Research, from the Ministry of Education, Culture, Sports, Science and Technology of Japan (Grant numbers 22129004, 24310144, and 26640122) (RK); (3) MEXT KAKENHI, Grant-in-Aid for Scientific Research (C) (Grant numbers 22790331 and 24510275) from the Ministry of Education, Culture, Sports, Science and Technology of Japan (AM); (4) MEXT KAKENHI, Grant-in-Aid for Scientific Research (C) (Grant number 24700371) from the Ministry of Education, Culture, Sports, Science and Technology of Japan (HH); (5) The Comprehensive Brain Science Network (Grant number 221S003), the Ministry of Education, Culture, Sports, Science and Technology of Japan (SM), and (6) National Center for Geriatrics and Gerontology Funds (Grant number 23-42), Obu, Japan (SM).

\section{Availability of data and materials}

The datasets generated and/or analyzed during the current study are not publicly available because they contain information that could compromise research participant privacy/consent, but are available from the corresponding author on reasonable request.

\section{Authors' contributions}

RK and $\mathrm{NH}$ conceived and designed the study. SM, HH, and YS participated in the neuropathological diagnosis of donor brains and the collection of brain tissue and serum. TI and KK participated in the clinical diagnosis of subjects and the collection of serum. NH performed the experiments. $\mathrm{NH}$ and $\mathrm{MK}$ analyzed the data. $\mathrm{NH}, \mathrm{RK}$, and TI wrote the manuscript. AM, HH, KK, MK, YS, and SM revised the manuscript. All authors read and approved the final manuscript.

\section{Competing interests}

The authors declare that they have no competing interests.

\section{Consent for publication}

Not applicable.

\section{Ethics approval and consent to participate}

This study was approved by the Institutional Review Board of Niigata University School of Medicine and Tokyo Metropolitan Geriatric Hospital and Institute of Gerontology. Written informed consent was obtained from each of the participants or the respective legal guardian. All the samples were anonymously analyzed.

\footnotetext{
Author details

'Department of Molecular Genetics, Bioresource Science Branch, Center for Bioresources, Brain Research Institute, Niigata University, Niigata, Japan. ${ }^{2}$ Department of Neuropathology, Tokyo Metropolitan Geriatric Hospital and Institute of Gerontology, Tokyo, Japan. ${ }^{3}$ Department of Pathology, National Center Hospital of Neurology and Psychiatry, Tokyo, Japan. ${ }^{4}$ Center for Transdisciplinary Research, Niigata University, Niigata, Japan. ${ }^{5}$ Asahigawaso Research Institute, Asahigawaso Medical-Welfare Center, Okayama, Japan. ${ }^{6}$ Department of Molecular Genetics, Brain Research Institute, Niigata University, 1-757 Asahimachi, Niigata 951-8585, Japan.
}

Received: 10 December 2016 Accepted: 26 January 2017 Published online: 31 January 2017

\section{References}

1. Absalon S, Kochanek DM, Raghavan V, Krichevsky AM (2013) MiR-26b, upregulated in Alzheimer's disease, activates cell cycle entry, tau-phosphorylation, and apoptosis in postmitotic neurons. J Neurosci 33:14645-59

2. Agarwal V, Bell GW, Nam JW, Bartel DP (2015) Predicting effective microRNA target sites in mammalian mRNAs. Elife 4:e05005

3. Anders S, Pyl PT, Huber W (2015) HTSeq-a Python framework to work with high-throughput sequencing data. Bioinformatics 31:166-9

4. Banzhaf-Strathmann J, Benito E, May S, Arzberger T, Tahirovic S, Kretzschmar $\mathrm{H}$ et al (2014) MicroRNA-125b induces tau hyperphosphorylation and cognitive deficits in Alzheimer's disease. EMBO J 33:1667-80

5. Bartel DP (2004) MicroRNAs: genomics, biogenesis, mechanism, and function. Cell 116:281-97

6. Bartel DP (2009) MicroRNAs: target recognition and regulatory functions. Cell 136:215-33

7. Benjamini $Y$, Hochberg $Y$ (1995) Controlling the false discovery rate: a practical and powerful approach to multiple testing. J R Statist Soc B 57:289-300

8. Blennow K, Hampel H, Weiner M, Zetterberg H (2010) Cerebrospinal fluid and plasma biomarkers in Alzheimer disease. Nat Rev Neurol 6:131-44

9. Bonda DJ, Lee HP, Kudo W, Zhu X, Smith MA, Lee HG (2010) Pathological implications of cell cycle re-entry in Alzheimer disease. Expert Rev Mol Med 12:e19

10. Braak H, Alafuzoff I, Arzberger T, Kretzschmar H, Del Tredici K (2006) Staging of Alzheimer disease-associated neurofibrillary pathology using paraffin sections and immunocytochemistry. Acta Neuropathol 112:389-404

11. Braak H, Braak E (1991) Neuropathological stageing of Alzheimer-related changes. Acta Neuropathol 82:239-59

12. Cheng L, Doecke JD, Sharples RA, Villemagne VL, Fowler CJ, Rembach A et al (2015) Prognostic serum miRNA biomarkers associated with Alzheimer's disease shows concordance with neuropsychological and neuroimaging assessment. Mol Psychiatry 20:1188-96

13. Chow HM, Herrup K (2015) Genomic integrity and the ageing brain. Nat Rev Neurosci 16:672-84

14. Crossland RE, Norden J, Bibby LA, Davis J, Dickinson AM (2016) Evaluation of optimal extracellular vesicle small RNA isolation and qRT-PCR normalisation for serum and urine. J Immunol Methods 429:39-49

15. Cummings $J \mathrm{~L}$ (2011) Biomarkers in Alzheimer's disease drug development. Alzheimers Dement 7:e13-44

16. da Huang W, Sherman BT, Lempicki RA (2009) Systematic and integrative analysis of large gene lists using DAVID bioinformatics resources. Nat Protoc 4:44-57

17. da Huang W, Sherman BT, Lempicki RA (2009) Bioinformatics enrichment tools: paths toward the comprehensive functional analysis of large gene lists. Nucleic Acids Res 37:1-13

18. Dobin A, Davis CA, Schlesinger F, Drenkow J, Zaleski C, Jha S et al (2013) STAR: ultrafast universal RNA-seq aligner. Bioinformatics 29:15-21

19. Frade JM, Ovejero-Benito MC (2015) Neuronal cell cycle: the neuron itself and its circumstances. Cell Cycle 14:712-20

20. Geekiyanage $H$, Chan C (2011) MicroRNA-137/181c regulates serine palmitoyltransferase and in turn amyloid $\beta$, novel targets in sporadic Alzheimer's disease. J Neurosci 31:14820-30

21. Geekiyanage $H$, Jicha GA, Nelson PT, Chan C (2012) Blood serum miRNA: noninvasive biomarkers for Alzheimer's disease. Exp Neurol 235:491-6

22. Hébert SS, Horré K, Nicolaï L, Papadopoulou AS, Mandemakers W, Silahtaroglu AN et al (2008) Loss of microRNA cluster miR-29a/b-1 in sporadic Alzheimer's disease correlates with increased BACE1/beta-secretase expression. Proc Natl Acad Sci U S A 105:6415-20

23. Henriksen K, O'Bryant SE, Hampel H, Trojanowski JQ, Montine TJ, Jeromin A et al (2014) The future of blood-based biomarkers for Alzheimer's disease. Alzheimers Dement 10:115-31

24. Hu Z, Zhao J, Hu T, Luo Y, Zhu J, Li Z (2015) miR-501-3p mediates the activity-dependent regulation of the expression of AMPA receptor subunit GluA1. J Cell Biol 208:949-59

25. Kirschner MB, Kao SC, Edelman JJ, Armstrong NJ, Vallely MP, van Zandwijk N et al (2011) Haemolysis during sample preparation alters microRNA content of plasma. PLoS One 6:e24145

26. Kozomara A, Griffiths-Jones S (2014) miRBase: annotating high confidence microRNAs using deep sequencing data. Nucleic Acids Res 42:D68-73

27. Kruman II, Wersto RP, Cardozo-Pelaez F, Smilenov L, Chan SL, Chrest FJ et al (2004) Cell cycle activation linked to neuronal cell death initiated by DNA damage. Neuron 41:549-61 
28. Kumar P, Dezso Z, MacKenzie C, Oestreicher J, Agoulnik S, Byrne M et al (2013) Circulating miRNA biomarkers for Alzheimer's disease. PLoS One 8:e69807

29. Kuwano R, Miyashita A, Arai H, Asada T, Imagawa M, Shoji M et al (2006) Dynamin-binding protein gene on chromosome $10 \mathrm{q}$ is associated with late-onset Alzheimer's disease. Hum Mol Genet 15:2170-82

30. Langmead B, Trapnell C, Pop M, Salzberg SL (2009) Ultrafast and memoryefficient alignment of short DNA sequences to the human genome. Genome Biol 10:R25

31. Lau P, Bossers K, Janky R, Salta E, Frigerio CS, Barbash S et al (2013) Alteration of the microRNA network during the progression of Alzheimer's disease. EMBO Mol Med 5:1613-34

32. Leidinger P, Backes $C$, Deutscher S, Schmitt K, Mueller SC, Frese $K$ et al (2013) A blood based 12-miRNA signature of Alzheimer disease patients. Genome Biol 14:R78

33. Li Y, Kowdley KV (2012) Method for microRNA isolation from clinical serum samples. Anal Biochem 431:69-75

34. Livak KJ, Schmittgen TD (2001) Analysis of relative gene expression data using real-time quantitative PCR and the 2(-Delta Delta C(T)) Method. Methods 25:402-8

35. Love Ml, Huber W, Anders S (2014) Moderated estimation of fold change and dispersion for RNA-seq data with DESeq2. Genome Biol 15:550

36. Lugli G, Cohen AM, Bennett DA, Shah RC, Fields CJ, Hernandez AG et al (2015) Plasma exosomal miRNAs in persons with and without Alzheimer disease: Altered expression and prospects for biomarkers. PLoS One 10:e0139233

37. Martin M (2011) Cutadapt removes adapter sequences from highthroughput sequencing reads. EMBnet 17:10-2

38. McKhann G, Drachman D, Folstein M, Katzman R, Price D, Stadlan EM (1984) Clinical diagnosis of Alzheimer's disease: report of the NINCDS-ADRDA Work Group under the auspices of Department of Health and Human Services Task Force on Alzheimer's Disease. Neurology 34:939-44

39. Mitchell PS, Parkin RK, Kroh EM, Fritz BR, Wyman SK, Pogosova-Agadjanyan EL et al (2008) Circulating microRNAs as stable blood-based markers for cancer detection. Proc Natl Acad Sci U S A 105:10513-18

40. Murayama S, Saito $Y$ (2004) Neuropathological diagnostic criteria for Alzheimer's disease. Neuropathology 24:254-60

41. Nunez-Iglesias J, Liu CC, Morgan TE, Finch CE, Zhou XJ (2010) Joint genome-wide profiling of miRNA and mRNA expression in Alzheimer's disease cortex reveals altered miRNA regulation. PLoS One 5:e8898

42. Sheinerman KS, Tsivinsky VG, Abdullah L, Crawford F, Umansky SR (2013) Plasma microRNA biomarkers for detection of mild cognitive impairment: biomarker validation study. Aging (Albany NY) 5:925-38

43. Smith PY, Hernandez-Rapp J, Jolivette F, Lecours C, Bisht K, Goupil C et al (2015) miR-132/212 deficiency impairs tau metabolism and promotes pathological aggregation in vivo. Hum Mol Genet 24:6721-35

44. Swerdlow RH (2012) Alzheimer's disease pathologic cascades: who comes first, what drives what. Neurotox Res 22:182-94

45. Tan L, Yu JT, Tan MS, Liu QY, Wang HF, Zhang W et al (2014) Genome-wide serum microRNA expression profiling identifies serum biomarkers for Alzheimer's disease. J Alzheimers Dis 40:1017-27

46. Wang WX, Rajeev BW, Stromberg AJ, Ren N, Tang G, Huang Q et al (2008) The expression of microRNA miR-107 decreases early in Alzheimer's disease and may accelerate disease progression through regulation of beta-site amyloid precursor protein-cleaving enzyme 1. J Neurosci 28:1213-23

47. Wang K, Yuan Y, Cho JH, McClarty S, Baxter D, Galas DJ (2012) Comparing the MicroRNA spectrum between serum and plasma. PLoS One 7:e41561

48. Wong HK, Veremeyko T, Patel N, Lemere CA, Walsh DM, Esau C et al (2013) De-repression of FOXO3a death axis by microRNA-132 and -212 causes neuronal apoptosis in Alzheimer's disease. Hum Mol Genet 22:3077-92

49. Zanutto S, Pizzamiglio S, Ghilotti M, Bertan C, Ravagnani F, Perrone F et al (2014) Circulating miR-378 in plasma: a reliable, haemolysis-independent biomarker for colorectal cancer. Br J Cancer 110:1001-7

\section{Submit your next manuscript to BioMed Central and we will help you at every step:}

- We accept pre-submission inquiries

- Our selector tool helps you to find the most relevant journal

- We provide round the clock customer support

- Convenient online submission

- Thorough peer review

- Inclusion in PubMed and all major indexing services

- Maximum visibility for your research

Submit your manuscript at www.biomedcentral.com/submit
C Biomed Central 